\title{
On-chip measurement of the Brownian relaxation frequency of magnetic beads using magnetic tunneling junctions
}

Donolato, M.; Sogne, E.; Dalslet, Bjarke Thomas; Cantoni, M.; Petti, D.; Cao, J.; Cardoso, F.; Cardoso, S.; Freitas, P. P.; Hansen, Mikkel Fougt

Total number of authors:

11

Published in:

Applied Physics Letters

Link to article, DOI:

$10.1063 / 1.3554374$

Publication date:

2011

Document Version

Publisher's PDF, also known as Version of record

Link back to DTU Orbit

Citation (APA):

Donolato, M., Sogne, E., Dalslet, B. T., Cantoni, M., Petti, D., Cao, J., Cardoso, F., Cardoso, S., Freitas, P. P., Hansen, M. F., \& Bertacco, R. (2011). On-chip measurement of the Brownian relaxation frequency of magnetic beads using magnetic tunneling junctions. Applied Physics Letters, 98(7), 073702. https://doi.org/10.1063/1.3554374

\section{General rights}

Copyright and moral rights for the publications made accessible in the public portal are retained by the authors and/or other copyright owners and it is a condition of accessing publications that users recognise and abide by the legal requirements associated with these rights.

- Users may download and print one copy of any publication from the public portal for the purpose of private study or research.

- You may not further distribute the material or use it for any profit-making activity or commercial gain

- You may freely distribute the URL identifying the publication in the public portal 


\title{
On-chip measurement of the Brownian relaxation frequency of magnetic beads using magnetic tunneling junctions
}

\author{
M. Donolato ${ }^{1, a)}$ E. Sogne, ${ }^{1}$ B. T. Dalslet, ${ }^{2}$ M. Cantoni, ${ }^{1}$ D. Petti, ${ }^{1}$ J. Cao, ${ }^{3}$ F. Cardoso, ${ }^{3}$ \\ S. Cardoso, ${ }^{3}$ P. P. Freitas, ${ }^{3}$ M. F. Hansen, ${ }^{2}$ and R. Bertacco ${ }^{1}$ \\ ${ }^{1}$ Dipartimento di Fisica, L-NESS, Politecnico di Milano, Via Anzani 42, 22100 Como, Italy \\ ${ }^{2}$ Department of Micro- and Nanotechnology, Technical University of Denmark, DTU Nanotech, \\ Building 345 East, DK-2800 Kongens Lyngby, Denmark \\ ${ }^{3}$ INESC Microsistemas e Nanotecnologias (INESC MN), Rua Alves Redol 9, 1000-029 Lisbon, Portugal
}

(Received 23 November 2010; accepted 21 January 2011; published online 15 February 2011)

\begin{abstract}
We demonstrate the detection of the Brownian relaxation frequency of $250 \mathrm{~nm}$ diameter magnetic beads using a lab-on-chip platform based on current lines for exciting the beads with alternating magnetic fields and highly sensitive magnetic tunnel junction (MTJ) sensors with a superparamagnetic free layer. The first harmonic out-of-phase component of the MTJ response gives the imaginary part of the magnetic bead susceptibility, which peaks at the Brownian relaxation frequency. This work paves the way to on-chip implementation of Brownian magnetorelaxometry in innovative "lab-on-a-bead" assays for biomolecular recognition. (C) 2011 American Institute of Physics. [doi:10.1063/1.3554374]
\end{abstract}

Biochip platforms using magnetoresistive sensors have been used in the last few years for biomolecular detection of analyte concentrations down to the femtomolar range. ${ }^{1,2}$ In the usual biodetection scheme, the magnetic particles labeling the target biological entity are detected when bound to the sensor surface through a specific biochemical assay and excited by a magnetic field generated externally ${ }^{1,3}$ or on-chip. ${ }^{4-6}$ Another approach is the Brownian relaxation detection scheme, where the dynamic rotation response of magnetic beads in suspension is used to detect biomolecules. In this method, the target species bind to the surface of the magnetic beads and cause a shift of the Brownian relaxation frequency $f_{B}$. Sensitivities down to the picomolar range have been demonstrated off-chip using superconducting quantum interference devices ${ }^{7}$ or induction coil magnetometers. ${ }^{8}$ Recently, Dalslet et al. ${ }^{4}$ demonstrated on-chip measurements of $f_{B}$ for different commercial magnetic beads using the selffield from planar Hall effect (PHE) sensors. In this paper, we use a different on-chip platform based on current lines (CLs) and highly sensitive magnetic tunnel junctions (MTJs) with a superparamagnetic free layer for measuring the $f_{B}$ of magnetic beads with a nominal diameter of $250 \mathrm{~nm}$. The suspension of magnetic beads is excited using the alternating magnetic field generated by CLs positioned close to the sensor area, while the measurements of the frequency behavior of the first harmonic of the MTJ output signal recorded using lock-in detection gives access to $f_{B}$.

MTJs with the structure (thicknesses in angstroms) glass $/ \mathrm{Ta}(50) / \mathrm{Ru}(180) / \mathrm{Ta}(30) / \mathrm{Pt}_{40} \mathrm{Mn}_{60}(200) / \mathrm{Co}_{90} \mathrm{Fe}_{10}$ (20) $/ \mathrm{Ru}(9) / \mathrm{Co}_{40} \mathrm{Fe}_{40} \mathrm{~B}_{20}(30) / \mathrm{MgO}(15) / \mathrm{Co}_{40} \mathrm{Fe}_{40} \mathrm{~B}_{20}(14.5) /$ $\mathrm{Ru}(50) / \mathrm{Ta}(50)$ were deposited by magnetron sputtering in a Nordiko 2000 system at INESC MN with a base pressure of $7 \times 10^{-9}$ Torr. $\mathrm{Co}-\mathrm{Fe}$ and $\mathrm{MgO}$ layers were deposited in rf mode and the other layers were deposited in dc mode, as described in Ref. 9. After deposition of the stack, devices were structured at L-NESS using optical lithography. Figure 1(a) shows the chip layout with eight MTJ sensors for

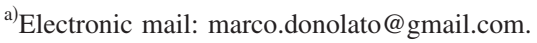

two-point electrical measurements and two parallel current lines (CLs). The junction areas were defined by ion milling in the form of rectangles $2.5 \mu \mathrm{m} \times 120 \mu \mathrm{m}$, where the shorter side $h$ is parallel to the easy axis, oriented along the $y$-axis in Fig. 1(a). After e-beam evaporation of $\operatorname{Cr}(50 \AA) /$ $\mathrm{Au}(1200 \AA)$ contacts, the samples were annealed at $330{ }^{\circ} \mathrm{C}$ at a pressure of $10^{-5}$ Torr for $1 \mathrm{~h}$ in a magnetic field of 400 $\mathrm{mT}$ applied along the positive $y$-direction. Then, a $2000 \AA$ thick layer of $\mathrm{SiO}_{2}$ was deposited in rf mode from an $\mathrm{SiO}_{2}$ target to electrically insulate the sensor stack. CLs made of $\mathrm{Cr}(50 \AA) / \mathrm{Au}(1500 \AA)$ were subsequently e-beam evaporated and defined by lift-off. Finally, a $5000 \AA$ thick protective coating of $\mathrm{Ta}_{2} \mathrm{O}_{5}$ was reactively sputter-deposited through a shadow mask. The width $w$ of the CLs is $6 \mu \mathrm{m}$ in the region close to the sensors, while it is $20 \mu \mathrm{m}$ away from sensors; the minimum edge-to-edge distance $d$ between the two CLs is $10 \mu \mathrm{m}$. On top of the chip was mounted a click-on microfluidic system. ${ }^{4}$ Figure 1(b) displays the sensor transfer curve versus field applied in the $y$-direction. These measurements were performed using a $1000 \mathrm{~Hz}$ bias current of amplitude $I_{s}=100 \mu \mathrm{A}$ injected in the MTJ via a Keithley 6221 current source and measuring the voltage signal with a Stanford Research Systems 830 lock-in amplifier connected in parallel to the current source. The curve is linear with magnetoresistance of $42 \%$ and a low-field sensitivity $S_{0}$ $=\left(R \mu_{0}\right)^{-1}\left(\mathrm{~d} R / \mathrm{d} H_{y}\right)=2.5 \% / \mathrm{mT}$. This sensitivity results from the combined effect of the shape anisotropy and the thickness of the $\mathrm{CoFeB}$ free layer in the superparamagnetic regime. ${ }^{9}$

In the linear region, the measured voltage $V$ over the MTJ sensor can be written as

$$
V=I_{s}\left(S_{0} \mu_{0}\left\langle H_{y}\right\rangle+R_{0}\right),
$$

where $\left\langle H_{y}\right\rangle$ is the total magnetic field in the $y$-direction averaged over the sensor area and $R_{0}$ is the sensor resistance at zero applied field. $\left\langle H_{y}\right\rangle$ has contributions from (i) a dc field $\left(H_{\mathrm{dc}}\right)$ being the sum of external fields and the demagnetizing field coming from the magnetic layers of the sensor itself, (ii) the field from the CLs, and (iii) the field from magnetic 

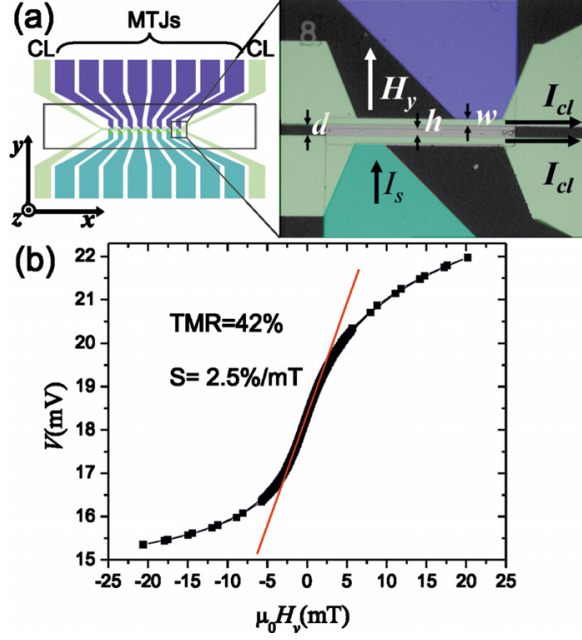

FIG. 1. (Color online) (a) Chip design $(7.5 \mathrm{~mm} \times 4.5 \mathrm{~mm})$. Each of the eight MTJ sensors is provided with two independent contacts for the bottom and top electrodes while there are two independent CLs. The larger rectangle indicates the outline of the fluid channel. An optical image of the MTJ sensor is shown on the right, with the direction of the currents in the CLs $\left(I_{\mathrm{CL}}\right)$, in the sensor $\left(I_{s}\right)$, and of the field $H_{y}$ produced by the CLs on the sensor plane. The CLs lie on top of the MTJ sensors, separated by $200 \mathrm{~nm}$ of $\mathrm{SiO}_{2}$. (b) Sensor transfer curve measured with $I_{s}=100 \mu \mathrm{A}$ at $1 \mathrm{kHz} ; R_{0}$ is $183 \Omega$.

beads near the sensor. We write the average field from the CLs on the sensor as $\beta_{0} I_{\mathrm{CL}}$, where $\beta_{0}$ depends on the device geometry and $I_{\mathrm{CL}}$ is the current injected in the CLs in the positive $x$-direction (cf. Fig. 1). Moreover, assuming a linear bead response, the average fields from the magnetic beads on the sensor are $\alpha \chi H_{\mathrm{dc}}$ and $\beta \chi I_{\mathrm{CL}}$, where $\chi$ is the effective bead susceptibility and $\alpha$ and $\beta$ are factors that depend on the sensor geometry and the bead size and distribution. ${ }^{10,11}$ Hence, we have

$$
\left\langle H_{y}\right\rangle=H_{\mathrm{dc}}+\alpha \chi H_{\mathrm{dc}}+\left(\beta_{0}+\beta \chi\right) I_{\mathrm{CL}} .
$$

When a magnetic bead is inserted in an ac magnetic field, its behavior is described by the complex magnetic susceptibility,

$$
\chi=\chi^{\prime}-\mathrm{i} \chi^{\prime \prime}=|\chi| \cos \phi-\mathrm{i}|\chi| \sin \phi,
$$

where $\chi^{\prime}$ and $\chi^{\prime \prime}$ are the in-phase and out-of-phase components with respect to the magnetic field, respectively, and $\phi$ is the phase lag of the magnetic bead response. In this work, the ac external field arises from the ac, $I_{\mathrm{CL}}(t)$ $=I_{\mathrm{CL}} \sin (2 \pi f t)$, injected in the parallel CLs, while a dc $I_{s}$ is injected in the MTJ and the signal $V$ is recorded using lock-in detection. Combining the previous equations, we calculate the first harmonic in-phase and out-of phase components $V_{1}^{\prime}$ and $V_{1}^{\prime \prime}$ as

$$
\begin{aligned}
& V_{1}^{\prime}=\frac{1}{\sqrt{2}} \mu_{0} S_{0} I_{s} I_{\mathrm{CL}}\left(\beta_{0}+\beta \chi^{\prime}\right), \\
& V_{1}^{\prime \prime}=-\frac{1}{\sqrt{2}} \mu_{0} S_{0} I_{s} I_{\mathrm{CL}} \beta \chi^{\prime \prime} .
\end{aligned}
$$

Hence, from these two components, it is possible to extract the complex magnetic susceptibility of the magnetic beads.

All measurements on bead suspensions were carried out on $\mathrm{NH}_{2}$-terminated Nanomag-D beads (Micromod, Rostock, Germany) with a nominal diameter of $250 \mathrm{~nm}$. The stock

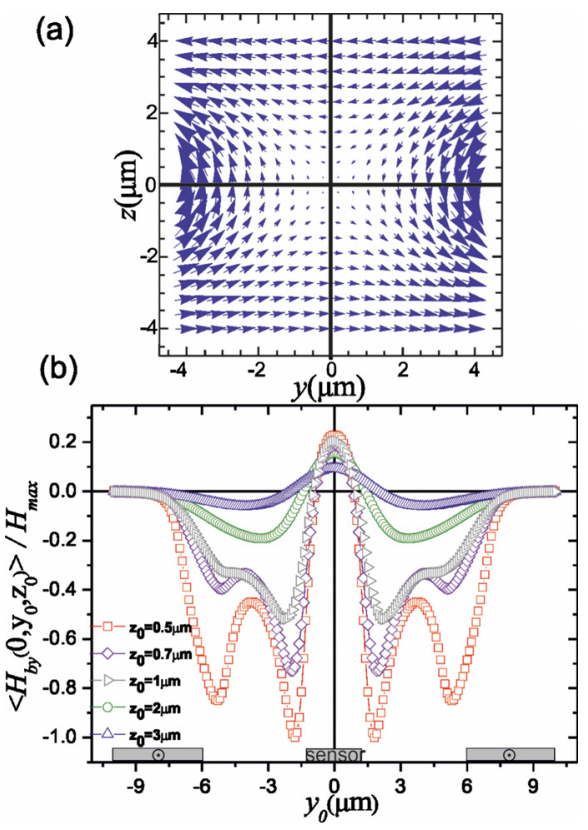

FIG. 2. (Color online) (a) Arrow plot in the $y-z$ plane of the magnetic field generated by a current flowing in the positive $x$-direction in the two CLs. (b) $\left\langle\mathbf{H}_{b y}\left(0, y_{0}, z_{0}\right)\right\rangle$ calculated in the sensor plane $\left(z_{s}=-200 \mathrm{~nm}\right)$ for a magnetic bead positioned at different heights $z_{0}$; the magnetic field is normalized to the modulus of the maximum average field $H_{\max }$ (calculated at a distance $\left.z_{0}=0.5 \mu \mathrm{m}\right)$

suspension was diluted with milli-Q water to a bead concentration of $0.3 \mathrm{mg} / \mathrm{ml}$.

For the investigated frequency window, Brownian relaxation dominates over Néel relaxation. The physical rotation of the magnetic beads is characterized by the Brownian relaxation frequency $f_{B}$ :

$$
f_{B}=\frac{k_{\mathrm{B}} T}{6 \pi \eta V_{h}},
$$

where $k_{\mathrm{B}}$ is the Boltzmann's constant, $T$ is the absolute temperature, $\eta$ is the dynamical viscosity of the fluid, and $V_{h}$ is the hydrodynamic volume of a bead. As theoretically described by Debye ${ }^{12}$ and Cole and Cole, ${ }^{13} \chi^{\prime}$ decreases monotonically with increasing frequency, whereas $\chi^{\prime \prime}$ peaks at $f=f_{B}$. Therefore, an analysis of $V_{1}^{\prime \prime}$ using the Cole-Cole expression ${ }^{13}$ can be used to extract information on the hydrodynamic radius of a population of suspended particles with a distribution of relaxation times.

Following an approach similar to that in Ref. 11 in order to estimate the constant $\beta$, we calculate the average magnetic field on the sensor area from a single bead in suspension above the sensor, magnetized by the field from infinitely long CLs. Considering the coordinate system of Fig. 1(a), the CLs are approximated with two-dimensional slabs lying in the $x-y$ plane that extend to infinity in the $x$-direction and with widths $w=6 \mu \mathrm{m}$ and edge-to-edge spacing $d=10 \mu \mathrm{m}$ in the $y$-direction. The sensor lies in a parallel plane at a distance $z_{s}=-200 \mathrm{~nm}$. Figure 2(a) shows the resulting magnetic flux density direction in the $y-z$ plane for $I_{\mathrm{CL}}>0$. As the sensor is placed at $z_{s}<0$, it is seen from Fig. 2 (a) that $\beta_{0}>0$. The normalized $y$-component of the average magnetic field on the sensor created by a single bead placed at $x_{0}=0$ and magnetized along $y$ for different heights $z_{0}\left\langle H_{b y}\left(0, y_{0}, z_{0}\right)\right\rangle$ is plotted as a function of its position on the $y$-axis in Fig. 2(b). If we consider a monolayer of beads of extension $2 d$ in the 

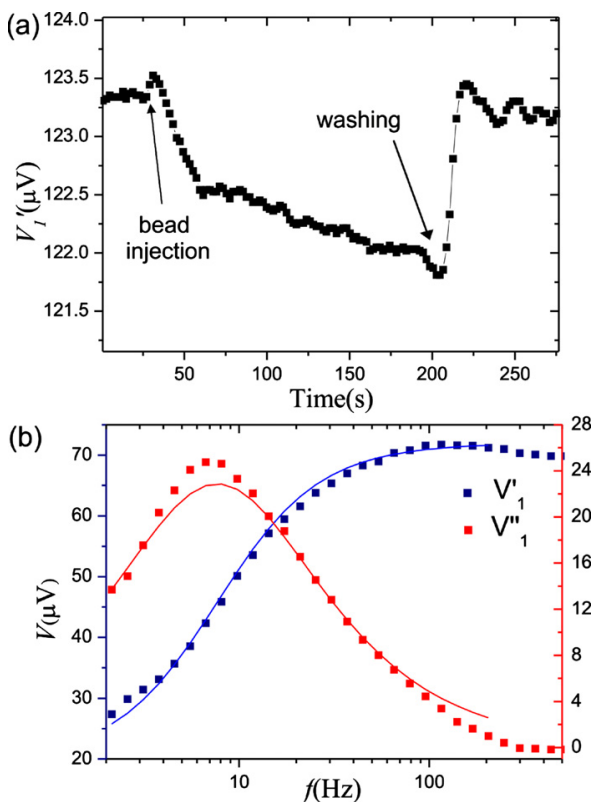

FIG. 3. (Color online) (a) $V_{1}^{\prime}$ vs time for $I_{\mathrm{CL}}=8 \mathrm{~mA}$ at $f=150 \mathrm{~Hz}$, $I_{s}=100 \mu \mathrm{A}$. A suspension of $250 \mathrm{~nm}$ Micromod Nanomag-D beads with concentration $0.3 \mathrm{mg} / \mathrm{ml}$ was injected in the fluidic channel after $35 \mathrm{~s}$ and washed after $210 \mathrm{~s}$. (b) Values of $V_{1}^{\prime}$ and $V_{1}^{\prime \prime}$ measured vs frequency $f$ of the current in the CLs for $I_{\mathrm{CL}}=12 \mathrm{~mA}$ and $I_{s}=100 \mu \mathrm{A}$. The solid lines are fits to the Cole-Cole expression.

$y$-direction, we can estimate $\beta \chi I_{\mathrm{CL}}$ by calculating the field created by a bead monolayer on the sensor plane

$$
\left\langle H_{m l, y}\left(z_{0}\right)\right\rangle=\frac{1}{2 d} \int_{-d}^{d}\left\langle H_{b y}\left(0, y_{0}, z_{0}\right)\right\rangle \mathrm{d} y_{0} .
$$

A bead at distance $z_{0}=3 \mu \mathrm{m}$ will give rise to $\left\langle H_{b y}\left(x_{0}, y_{0}, z_{0}\right)\right\rangle>0$ when it is above the sensor area and $\left\langle H_{b y}\left(x_{0}, y_{0}, z_{0}\right)\right\rangle<0$ when it is outside the sensor area. In a homogeneous field, the positive and negative contributions cancel each other, implying that in this case a monolayer of beads would not give a detectable sensor response. ${ }^{10}$ However, in the inhomogeneous magnetic field from the CLs, which is strongest over the CLs, the negative contribution from beads near the CLs outweighs the weaker positive contributions from beads over the sensor area when $z_{0} \leq 3 \mu \mathrm{m}$, as shown in Fig. 2(b). As a result, $\left\langle H_{m y}\left(z_{0}\right)\right\rangle$ is a net measurable field in the negative $y$-direction for $0<z_{0}<3 \mu \mathrm{m}$, corresponding to $\beta<0$.

The time evolution of $V_{1}^{\prime}$ for a dc in the sensor $I_{s}$ $=100 \mu \mathrm{A}$ and an ac in the CLs with $I_{\mathrm{CL}}=8 \mathrm{~mA}$ and $f$ $=200 \mathrm{~Hz}$ is shown in Fig. 3(a). In the absence of beads, the signal is proportional to the applied field. When a suspension of beads is introduced in the microfluidic system, the signal decreases, confirming that $\beta$ is negative. When the beads are washed away, the signal returns to its initial value. The very same CLs can be used to attract beads on the sensor area by injecting opposite currents in the lines to increase the gradient of the magnetic field and hence the capture force. After application of $10 \mathrm{~mA}$ in the CLs in the antiparallel configuration for $30 \mathrm{~min}$, we performed magnetorelaxometry experiments by injecting a dc $I_{s}=100 \mu \mathrm{A}$ in the sensor while sweeping the frequency $f$ of the ac $\left(I_{\mathrm{CL}}=12 \mathrm{~mA}\right)$ and measuring the corresponding values of $V_{1}^{\prime}$ and $V_{1}^{\prime \prime}$. Figure 3(b) shows the results of the frequency sweep between 2 and 500
Hz. As $S_{0}, I_{\mathrm{CL}}$, and $\beta_{0}$ are positive, while $\beta$ is negative, the data are consistent with Eq. (4) and with the frequency dependence of the susceptibility due to Brownian magnetic dynamics: $V_{1}^{\prime}$ depends linearly on $\chi^{\prime}$ and decreases with increasing values of $\chi^{\prime}$ and hence shows a minimum at low frequencies, while $V_{1}^{\prime \prime}$ is proportional to $\chi^{\prime \prime}$ and hence shows a positive maximum at $f=f_{B}$. The data were fitted using the Cole-Cole model [full lines in Fig. 3(b)]. The same set of parameters with a single scaling factor was used to fit the in-phase and out-of phase components of the signal, leading to $f_{B}=7.76 \mathrm{~Hz}$ and $\alpha=0.12$, where $\alpha$ is the Cole-Cole parameter. This corresponds to a hydrodynamic bead diameter of $d_{h}=397 \mathrm{~nm}$, which is higher than the nominal bead diameter, most likely due to bead-surface and bead-bead interactions as previously discussed for PHE sensors. ${ }^{4}$ The lower value of $d_{h}$ obtained using MTJ sensors with CLs compared to that obtained using the self-field PHE sensors $(420 \mathrm{~nm})$ is probably due to the reduced magnetostatic field from the MTJ structure and the lower sensor bias current, which results in a weaker attraction of the beads to the sensor surface.

In conclusion, we have demonstrated the combined use of MTJ sensors and on-chip CLs for measuring the Brownian relaxation frequency of magnetic beads. The negative variation of the sensor signal caused by the presence of the magnetic beads has been explained through analytical calculations. Due to the focusing action of the CLs on the sensor area and the high sensitivity of the MTJs, magnetorelaxometry spectra were obtained after only $30 \mathrm{~min}$ from the injection of the beads in the microfluidic system (instead of $5 \mathrm{~h}$ sedimentation time required in previous experiments employing PHE sensors without $\mathrm{CLs}^{4}$ ). This works paves the way to the development of a quantitative biodetection scheme employing MTJ sensors, which is the topic of our ongoing work.

This work was supported by Fondazione Cariplo via the project SpinBioMed (Project No. 2008.2330). M.F.H. and B.T.D. acknowledge the Danish Council for Independent Research, Technology and Production Sciences (Project No. 09-062257).

${ }^{1}$ V. C. Martins, F. A. Cardoso, J. Germano, S. Cardoso, L. Sousa, M. Piedade, P. P. Freitas, and L. P. Fonseca, Biosens. Bioelectron. 24, 2690 (2009).

${ }^{2}$ R. S. Gaster, D. A. Hall, C. H. Nielsen, S. J. Osterfeld, H. Yu, K. E. Mach, R. J. Wilson, B. Murmann, J. C. Liao, S. S. Gambhir, and S. X. Wang, Nat. Med. 15, 1327 (2009).

${ }^{3}$ S. X. Wang and G. Li, IEEE Trans. Magn. 44, 1687 (2008).

${ }^{4}$ B. T. Dalslet, C. D. Damsgaard, M. Donolato, M. Strømme, M. Strömberg, P. Svedlindh, and M. F. Hansen, Lab Chip 11, 296 (2011).

${ }^{5}$ B. M. de Boer, J. A. H. M. Kahlman, T. P. G. H. Jansen, H. Duric, and J. Veen, Biosens. Bioelectron. 22, 2366 (2007).

${ }^{6}$ L. Lagae, R. Wirix-Speetjens, J. Das, D. Graham, H. Ferreira, P. P. F. Freitas, G. Borghs, and J. De Boeck, J. Appl. Phys. 91, 7445 (2002).

${ }^{7}$ M. Strömberg, J. Göransson, K. Gunnarsson, M. Nilsson, P. Svedlindh, and M. Strømme, Nano Lett. 8, 816 (2008).

${ }^{8}$ A. P. Astalan, F. Ahrentorp, C. Johansson, K. Larsson, and A. Krozer, Biosens. Bioelectron. 19, 945 (2004).

${ }^{9}$ P. Wiśniowski, J. M. Almeida, S. Cardoso, N. P. Barradas, and P. P. Freitas J. Appl. Phys. 103, 07A910 (2008).

${ }^{10}$ C. D. Damsgaard and M. F. Hansen, J. Appl. Phys. 103, 064512 (2008).

${ }^{11}$ T. B. G. Hansen, C. D. Damsgaard, B. T. Dalslet, and M. F. Hansen, J. Appl. Phys. 107, 124511 (2010).

${ }^{12}$ P. Debye, Polar Molecules (Chemical Catalogue, New York, 1929).

${ }^{13}$ K. S. Cole and R. H. Cole, J. Chem. Phys. 9, 341 (1941). 